\title{
La prueba PISA: una mirada alternativa desde la Teoría del Actor-Red
}

\section{The PISA test: an Actor-Network Theory alternative approach}

\author{
Paola Paredes Suzarte ${ }^{1}$ \\ Universidad de Santiago de Chile, Santiago, Chile
}

(Rec: febrero 2015 - Acept: junio 2015)

\begin{abstract}
Resumen
En el marco de la proliferación de las evaluaciones estandarizadas, inscritas en lo que ha sido conceptualizado por la literatura como políticas de accountability o de rendición de cuentas, se ofrece por medio de este artículo una línea de pensamiento y discusión alternativa sobre la prueba PISA ${ }^{2}$. Utilizando como enfoque la Teoría del Actor-Red (TAR) de Bruno Latour, se presentan ejercicios de traslación epistémica de la prueba PISA desde un modelo de pensamiento positivista moderno, a uno materialista pragmático en el que se privilegia una descripción constructivista y analítica de los componentes que la constituyen. En base al seguimiento y análisis de noticias sobre la prueba PISA desplegadas en diarios electrónicos en Chile y Argentina, más la revisión de fuentes secundarias, se exponen algunas de las controversias suscitadas en torno a la evaluación y se discute sobre su posible impacto en nuestras percepciones sobre educación y calidad. También se problematiza el carácter neutral y objetivo de la prueba, y se levanta la pregunta acerca de su posible agencia en la cristalización de las relaciones de centro-periferia entre países que, en el marco del enfoque de la TAR, tiene sentido explorar.

Palabras clave: Prueba PISA, Teoría del Actor Red (TAR), Constructivismo.
\end{abstract}

\begin{abstract}
In the context of the proliferation of standardized testing, circumscribed by what has been conceptualized in the literature as "accountability policies", the aim of this paper is to offer an alternative line of thought and discussion on the PISA test. Using the approach of Bruno Latour's actor-network theory (ANT), I present exercises on epistemic translation of the PISA test from a modern-positivist model of thought to a materialistic-pragmatic one, in which a constructivist and analytical description of its constituent components is preferred. Based on the monitoring and analysis of news reports about PISA in electronic newspapers in Chile and Argentina, plus the review of secondary sources, I describe some of the controversies surrounding the test and discuss its potential impact on our perceptions of education and quality. Additionally, the allegedly neutral and objective character of the test is problematized, and it is raised the question of its possible agency in the crystallization of centerperiphery relations between countries which, in the framework of the ANT approach, makes sense to explore. Keywords: PISA test, Actor-Network Theory (ANT), constructivism.
\end{abstract}

\footnotetext{
1 Correspondencia a: Paola Paredes Suzarte. Universidad de Santiago de Chile, Departamento de Historia, Av. Ecuador \#3363, Estación Central, Región Metropolitana de Santiago, Chile. E-mail: giparedes@ hotmail.com.

2 Programme for International Student Assessment (PISA). La prueba PISA es una encuesta internacional trienal de carácter muestral que busca evaluar conocimiento y habilidades en de alumnos de 15 años de edad en las áreas de lenguaje, matemáticas y ciencias.
} 


\section{Introducción}

En la discusión presente en la Teoría del Actor-Red (TAR en adelante), se problematizan los supuestos epistémicos modernos en los que se generan una serie de pares dicotómicos y asimétricos entre sí. Ejemplo de ello es la dicotomía entre humano y no humano, sujeto-objeto, de la que se critica el hecho de que se les atribuya subjetividad a los primeros y objetividad y neutralidad a los segundos (Latour, 2007). En la misma línea se discute también que se les asigne a los primeros un estado de existencia, y con ello de agencia, en la conformación y mantención de eventos sociales mientras que a los segundos no, generando con eso la asimetría descrita.

En este marco de pensamiento entonces, se problematiza la dicotomía y asimetría producidas bajo un modo de pensar moderno, argumentando que no se observan al momento de estudiar un evento social, sino que son más bien una construcción conceptual moderna sin correlato en la materialidad, de ahí que el modo de pensar no moderno descrito por la TAR sea entendido como un modo de pensamiento materialista pragmático, que busca describir tanto a sujetos como objetos en las relaciones que establecen entre sí, asignándoles a ambos una agencia simétrica en la conformación y mantención de eventos sociales.

Así, la TAR desde un paradigma de pensamiento materialista pragmático plantea que como investigadores de lo social debemos seguir a los actantes ${ }^{3}$, por igual y simétricamente, para observar y describir su construcción y los recorridos materiales que junto a otros actantes establecen formando la red o el ensamblado socio-técnico ${ }^{4}$ que da origen y mantenimiento a ciertos fenómenos sociales. De Ahí que, bajo estas concepciones epistémicas, sea necesario seguir el rastro de los actantes y la evidencia empírica que van dejando en su recorrido, y que tenga sentido en nuestro caso preguntarse por la objetividad de PISA en lugar de atribuírsela por gracia, sólo por el hecho de ser un objeto técnico, sin explorar la evidencia. De esta forma, en lugar de asumir la prueba PISA exclusivamente como un dispositivo técnico y neutral, cobra sentido darse el espacio para explorar las evidencias empíricas dejadas

El concepto se toma de la semiótica para incluir a los no humanos en la discusión de lo social, reemplazando al de actor que sólo da cuenta de los humanos (Latour, 2001).

4 Se refiere a las asociaciones entre humanos y no humanos que se establecen en lo social. Dichas asociaciones dan vida y mantención a eventos sociales sin ser estables en sí; pueden variar generando cambios en lo social o pueden mantenerse ensambladas haciendo perdurable ciertos fenómenos. por ella desde el momento en que fue posible pensarla y validarla, preguntándose por su construcción y por las concepciones que transporta sobre educación, competencias, evaluación, etc. En este sentido, se espera encontrar, en dicha construcción, relaciones simétricas entre elementos técnicos, políticos, científicos, culturales, ideológicos, etc.

La prueba PISA en el marco de esta discusión será entendida como un cuerpo de asociaciones políticas y de gestión que la subjetivan, por cuanto aquí se entenderá que no existe división entre la ciencia y tecnología y la gestión social para generarla (Bijker, 1997; Callon \& Law, 1998; Knorr, 1995; Latour, 1999), yendo aún más allá para argumentar que estas mismas asociaciones son las que la robustecen ontológicamente ${ }^{5}$ (Knorr, 1995; Latour, 1999).

En el marco de esta discusión propuesta no se intenta invalidar o deslegitimizar la prueba PISA, sino que más bien se busca sacarla de su condición de caja negra ${ }^{6}$ y de tecnología neutral ${ }^{7}$. En definitiva, se persigue conocerla y entenderla mejor y más relacionalmente, preguntándole por su rol en las diferencias y jerarquización entre países, que desde esta teoría y paradigma pragmático resulta pertinente y necesaria. Teniendo presente que los eventos técnico-sociales suelen tener efectos positivos y negativos a la vez, se pretende discutir sobre la prueba PISA para plantear la necesidad de sacarla de su estatus de objeto neutral y sin agencia, de caja negra, en la que no tiene sentido explorarla en términos pragmáticos por cuanto su neutralidad y objetividad le son inherentes, para transportarla a un paradigma en que cobra relevancia su análisis para describir su construcción y los agentes involucrados en ella.

\section{Ejercicios de desplazamiento analítico de PISA}

Teniendo presente la discusión desplegada en la introducción de este trabajo, en el presente apartado se procederá a describir la manera en que la visualización

\footnotetext{
5 Por nivel ontológico se entiende el grado de relevancia y repercusión social. Ver Latour (2001), Capítulo 3: El flujo sanguíneo de la ciencia en La esperanza de Pandora.

6 Concepto tomado de la sociología de la ciencia que se refiere al hecho de que se sabe poco acerca de la construcción y funcionamiento de ciertos elementos técnicos y científicos en la medida en que funcionen bien y adquieran relevancia, tomándose de ellos solo los datos de entrada y salida.

7 Ver ejemplo sobre la inteligencia científica de Joliot, en $L a$ Esperanza de Pandora (Latour, 2001).
} 
y análisis de la prueba PISA suele cambiar según el marco epistémico desde el cual se piensa. El objetivo es problematizar las miradas totalizantes y absolutistas que no dan cabida a la formulación de preguntas que, en el marco de la TAR, tiene sentido formular para llegar a tener una comprensión más acabada y compleja sobre los objetos de estudio (Haraway, 1988, 1991; Latour, 1999).

\section{Ejercicio I: abrir la caja negra}

En un paradigma moderno, como ya ha sido señalado, no es necesario preguntarse por la historia del organismo internacional que dio vida a la prueba PISA $(\mathrm{OCDE})^{8}$, por cuanto las prácticas de su producción son circunscritas a la esfera técnica-científica distinta e independiente de la esfera socio-económica en la que se halla inscrita la OCDE. Dicho de otro modo, la OCDE no es relevante ni necesita ser explorada, porque al instaurar la separación entre el objeto PISA (actante no humano/objetivo) y la organización socio-política que participa de su constitución (actante humano/subjetivo), la prueba PISA pasa a existir de manera independiente adquiriendo el estatus de objeto técnico que cuenta con una validez intrínseca. De este modo, la prueba PISA es tomada como una unidad cerrada e independiente de la cual no se necesita saber sobre su procedencia ni sobre sus procesos de construcción para confiar en los datos que arroja, dada su neutralidad y estandarización técnica, adquiriendo así su calidad de caja negra.

Sin embargo, al trasladar la prueba PISA a un paradigma donde no se establece la escisión entre objeto y sujeto por cuanto se le reconoce a ambos un nivel performativo simétrico en el evento social y de co-constitución entre sí, la OCDE es entendida como parte indisociable de PISA y por ende, determinante en las concepciones sobre educación y habilidades transportadas por la prueba, entre otras cosas. Es por ello que se vuelve relevante conocer su historia, los procesos de su construcción y la ideología que se halla tras sus líneas de acción.

Al preguntarse por los orígenes y por los fines de la OCDE es posible encontrar una breve descripción de ambas en su página web que a continuación cito:

Fundada en 1961, la Organización para la Cooperación y el Desarrollo Económicos (OCDE) agrupa a 34 países miembros y su misión es promover políticas que mejoren el bienestar

\footnotetext{
8 Organización para la Cooperación y el Desarrollo Económico.
}

económico y social de las personas alrededor del mundo.

La OCDE ofrece un foro donde los gobiernos puedan trabajar conjuntamente para compartir experiencias y buscar soluciones a los problemas comunes. Trabajamos para entender que es lo que conduce al cambio económico, social y ambiental. Medimos la productividad y los flujos globales del comercio e inversión. Analizamos y comparamos datos para realizar pronósticos de tendencias. Fijamos estándares internacionales dentro de un amplio rango de temas de políticas públicas (Organización para la Cooperación y el Desarrollo Económico [OCDE], 2015, párr. 1-2)

Tener presente el contexto en el que surge la OCDE, en conjunto con sus objetivos y lineamientos, nos ayuda a entender de manera más acabada la prueba PISA, por cuanto es posible comprender, a partir de esa información, la racionalidad que se halla tras la prueba que es lo que en definitiva determina su agencia, entendiendo por ésta su finalidad y el recorrido que establece para lograr su propósito y su efecto.

$\mathrm{Al}$ considerar que la OCDE fue fundada 16 años después del fin de la II guerra mundial, en un contexto social y político tensionado por la devastación generada por la guerra, y con el objeto de ejecutar el Plan Marshall ${ }^{9}$, se entiende y visualiza mejor que su agenda central sea velar por el desarrollo económico en el marco de una sociedad global y capitalista. Es por ello que una de sus principales líneas de acción es propiciar políticas que promuevan el bienestar económico y social de las personas (Brinek \& Hopmann, 2007; Dolin, 2007; Leibfried \& Martens, 2009; OCDE, 2015).

Lo anterior, como ya ha sido mencionado, es relevante en un enfoque TAR por cuanto describe las concepciones que se hallan detrás de las líneas de acción de la OCDE que son parte de la racionalidad con que se dio vida a la prueba PISA. En ese sentido se puede pensar desde la TAR que la prueba PISA transporta en si una agencia economicista, en el sentido de que busca por medio del levantamiento de información, guiar y orientar la formulación de políticas públicas destinadas al mejoramiento de la calidad educativa. Por lo tanto, es concebida por la OCDE como un pilar fundamental para asegurar el crecimiento y bienestar económico de los países (OCDE, 2006, 2014; Pedró, 2012).

\footnotetext{
9 Inicialmente European Recovery Program, ERP. Ayuda financiera otorgada por EEUU a Europa con el fin a apoyar su recuperación económica.
} 
Con relación a esto último, han surgido durante los últimos años, críticas en torno a la prueba PISA provenientes principalmente desde el mundo académico, que no se hallan necesariamente circunscritas en torno a la trama de discusión en educación, sino que provienen de distintas áreas de producción del conocimiento entre las que se destacan aquellas provenientes de los estudios sobre políticas públicas y sobre Ciencia, Tecnología y Sociedad, CTS.

A modo de ejemplo, me gustaría presentar algunas de las críticas o reflexiones en torno a la prueba PISA, elaboradas y difundidas por académicos a través de medios de comunicación masiva. La primera de ellas son las desarrollas en la Declaración del Grupo de Trabajo (GT) $\mathrm{CLACSO}^{10}$ de Políticas educativas y derecho a la educación en América Latina y el Caribe, y la segunda, son problematizaciones que han sido levantadas en un marco internacional por un conjunto de académicos que elaboraron una carta dirigida a Andreas Schleicher, director del programa PISA de la OCDE y publicada en The Guardian ${ }^{11}$.

El grupo de trabajo de Políticas educativas y derecho a la educación en América Latina y el Caribe, tras analizar las propuestas que plantean los operativos estandarizados de evaluación como clave para el mejoramiento de la educación, elaboró una carta titulada "No a PISA. Por una evaluación al servicio de una educación emancipadora", en la que se señalan, entre otras, algunas de las críticas que citaré:

a. Su condición de dispositivo "único e inapelable de medición", homogéneo, estandarizado, que opera propiciando la competencia, clasificación y rotulación jerarquizante a partir de la publicidad de los puntajes.

b. Como lo muestran múltiples investigaciones, la aplicación del dispositivo está provocando la uniformización de las prácticas docentes en vistas a lograr un mejor desempeño en las pruebas.

c. La mercantilización que supone la creación de un dispositivo gestionado globalmente, en tanto profundiza en una lógica de financiamiento educativo transnacional en clave de lucro. El círculo se cierra con la tercerización del diseño y aplicación de la evaluación a través de la contratación de agencias privadas.

d. La asociación entre la aplicación de las pruebas y la evaluación de la calidad de la educación, en tanto el concepto de calidad educativa no es unívoco;

\footnotetext{
10 Consejo Latino Americano de Ciencias Sociales

11 Diario de UK ganador del premio Pulitzer.
}

está inserto en una red compleja de significación histórica, social y política.

e. Cuestionamos también la validez de las pruebas PISA y sus resultados para conocer y resolver los problemas de la escuela pública en América Latina y El Caribe preocupada por asegurar el derecho a la educación (Consejo Latinoamericano de Ciencias Sociales [CLACSO], 2014).

Por otro lado, los académicos firmantes en la carta dirigida a Andreas Schleicher y publicada en The Guardian, expresan también su preocupación en aspectos que están en sintonía con los levantados por el GT de CLACSO, de los cuales procederé a citar sólo algunos, de modo ilustrativo:

a. A pesar de los cuestionamientos sobre su validez, la evaluación estandarizada ha sido utilizada en varias naciones y desde hace décadas, sin embargo, PISA ha provocado una escalada de su uso. Por ejemplo, en Estados Unidos PISA ha sido evocada como la mayor justificación para el programa Race to the top que a intensificado la medición estandarizada que rankea y etiqueta a alumnos, profesores y administrativos.

b. La OCDE como una organización de cooperación para el desarrollo económico, está naturalmente sesgada a favor del rol de la educación pública como motor de la economía. Pero, preparar a los alumnos para un empleo lucrativo no es la principal meta de la educación pública sino preparar a los alumnos para la participación en democracia y para un desarrollo, crecimiento y bienestar personal.

c. A diferencia de organizaciones de Naciones Unidas tales como UNESCO o UNICEF que tienen mandatos claros y legítimos para mejorar la educación y las vidas de los niños en todo el mundo, la OCDE no tiene tal mandato. Tampoco existen a la fecha mecanismos para una efectiva participación democrática en sus procesos de toma de decisión en torno a la educación.

d. Para sacar adelante PISA y sus servicios de seguimiento, la OCDE ha acogido "alianzas público-privadas" y se ha aliado con compañías multinacionales con fines de lucro listas para ganar financieramente de los déficits -reales o percibidosdestapados por PISA. Algunas de estas compañías proveen servicios educativos a escuelas y distritos educativos de Estados Unidos, de manera masiva y con fines de lucro, y tienen planes para desarrollar una educación privada con fines de lucro en África, 
donde la OCDE está planeando introducir PISA (OCDE \& PISA, 2014).

En suma, al abrir la prueba PISA y explorar su construcción considerando, entre otros aspectos, la institución que le dio vida, las racionalidades que tiene tras sí y el modo en que se aplica, la prueba comienza a ser subjetivada y relativizada, incluso en términos de validez (Dolin, 2007; Pedró, 2012). Dicha relativización queda de manifiesto en las críticas citadas en donde la prueba PISA deja de ser un dispositivo desconocido desde el cual sólo se toman los datos para generar políticas públicas con todo lo que eso implica en términos de impacto sobre los países y sus ciudadanos, y en este caso, más particularmente, sobre los alumnos y alumnas que se hallan en el sistema escolar.

\section{Ejercicio II: preguntarse por la agencia del} objeto PISA en la conformación y mantención de relaciones de dependencia

En Reensamblar lo social, Latour (2005) refiriéndose al trabajo de Shirley Strum ${ }^{12}$ sobre los mandriles, señala como las tecnologías ayudan eficientemente a mantener las jerarquías que se establecen en el orden social, cuestión que en este marco de pensamiento justifica el hecho de preguntarse por la agencia de la prueba PISA, como tecnología, en la estratificación entre países. Shirley Strum, por medio de su trabajo, da cuenta de que los mandriles al no contar con tecnologías como las nuestras, tienen que invertir todos los días una enorme cantidad de esfuerzo en torno a interacciones cara a cara, como peleas y rituales, para mantener el ordenamiento que han construido, también en base a esas interacciones de jerarquización y dominio. En el citado trabajo, señala al respecto, que dicha ausencia de tecnologías son las que provocan que su ordenamiento de estratificación se constituya como un entramado débil que necesita ser reforzado frecuentemente por medio de las interacciones descritas.

A partir de lo anterior es posible extrapolar que nosotros al tener tecnologías, invertimos menos esfuerzo en la constitución y mantenimiento de las diferencias porque éstas ayudan a generar y fijar un tipo de ordenamiento jerarquizante. Desde este punto de vista, cobra sentido pensar que PISA podría estar construyendo las diferencias, por ejemplo, por medio de

\footnotetext{
12 Véase Strum, S., Almost Human: A Journey into the World of Badoons, Random Hause, Nueva York, 1987., Strum, S. y Latour, B., The Meanings of Social: From Baboons to Human, en "Information sur les Sciences Sociales/Social Science Information, 26, (1987), pp. 783-802.
}

sesgos transportados por las preguntas en sí o por medio de la traducción que se hace de ellas (Gorur, 2011; Puchhammer, 2007), o bien simplemente, porque ante la imposibilidad de ser neutral, termina favoreciendo a ciertos países en desmedro de otros (Bodin, 2007). De igual modo, se puede señalar que la prueba PISA por medio de los rankings y de su difusión mediática, fija las diferencias que construye, dándoles notoriedad, o lo que en términos teóricos vendría a ser entendido como un nivel ontológico mayor, haciendo existir las diferencias a nivel público y en el imaginario social (Pedró, 2012).

Latour (1998) argumenta que para entender las relaciones de poder hay que comprender el rol que los actantes no humanos juegan en ellas, puesto que son éstos los que mantienen la sociedad unida y duradera en su ordenamiento. De este modo, y a diferencia de los mandriles, nosotros sí podemos atribuirle agencia a tecnologías como la prueba PISA en la creación y fijación de ordenaciones de subordinación. En el caso de la prueba PISA podemos señalar que al mismo tiempo que constituye a los países del norte como superiores a los del sur, en función del dictamen que establece sobre la calidad de sus sistemas educativos, se constituye a sí misma como un ente descriptor y predictor imprescindible para lograr mejorar los sistemas educativos a imagen y semejanza de aquellos que rinden bien, junto con situar a la OCDE en una posición de poder prescriptivo.

La prueba PISA, inscrita en la lógica de las políticas de responsabilización que tienen tras de sí una racionalidad de mercado, transporta la idea de que los actores son responsables de sus propios logros y que deben dar cuenta de ellos, en conjunto con la idea de que la competencia (ranking) estimula y facilita la superación (Falabella, 2014; Gorur, 2011; Leach et al., 2007). Teniendo presente lo anterior, es posible argumentar que a partir de dichas concepciones liberales ya instauradas, emerge con éxito la expansión territorial de la prueba PISA en su forma de ranking. De este modo el éxito de la prueba PISA podría deberse al hecho de que encuentra resonancia en un ensamblado transinstitucional ya existente, que ha sido conceptualizado por Foucault como gubernamentalidad ${ }^{13}$ (Foucault, 2002, 2005, 2006, 2007, 2009, 2012).

Volviendo a nuestro caso de estudio (y a modo de cierre de este apartado), se sostendrá que la OCDE al posicionarse a través de su co-constitución con la prueba PISA como un ente prescriptor de políticas

\footnotetext{
13 Ver los cursos de 1978 y 1979 en el Collège de France.
} 
educativas, por medio de la evaluación y ranking que establece entre países, fija las relaciones de centroperiferia al dictaminar desde su particularismo y/o eurocentrismo, la calidad de la educación de países cultural y económicamente diversos ofreciendo al mismo tiempo orientaciones (desde el centro) para mejorar.

\section{Ejercicio III: rastrear en los diarios las controversias desplegadas en torno a PISA}

Prestar atención a las controversias es una de las tareas más primordiales estipuladas dentro de la metodología de la TAR, porque es la manera de hacer rastreable lo social (Latour, 2008). De hecho, Latour en su libro Nunca fuimos modernos (2007), comienza citando las páginas de un diario para ilustrar que, por medio de las controversias desplegadas en él, además de identificar a los actantes involucrados, se puede notar el carácter híbrido de los objetos o fenómenos en cuestión, es decir, notar que en un simple objeto o evento hay muchos otros objetos o eventos de distintas esferas que lo constituyen. Al seguir las noticias en torno a PISA, queda de manifiesto que no es sólo una evaluación internacional, sino una asociación entre técnicos, políticos, expertos, tecnologías, ideologías, etc. (Gorur, 2011).

Prestar atención a las controversias, es además, un paso metodológico que pone en práctica las enunciaciones epistémicas de la teoría, por cuanto permite acercarse a un evento social para describirlo y seguirlo en su devenir fluido, sin cristalizarlo como se suele hacer en el modo de pensar moderno al entrar con categorías conceptuales predefinidas que podrían sesgar y hasta determinar lo que se observa (Haraway, 1988, 1989).

Teniendo presente lo anterior, por medio de la generación de una alerta en Google en Agosto de 2013, he estado rastreando por más de dos años las noticias en torno a PISA en Chile y Argentina. Por medio de la lectura y análisis de alrededor de 2.500 noticias publicadas en diarios electrónicos de Chile y Argentina, he podido establecer categorías emergentes ${ }^{14}$ que me han permitido, además de identificar el carácter híbrido de PISA y a los actantes involucrados en ella, visualizar en términos cuantitativos y cualitativos que el grado de validación de los resultados arrojados por la prueba, difiere considerablemente entre ambos países. Utilizando el constructo conceptual de la teoría del Actor-Red, se podría sostener que en Chile se le otorga más validez

\footnotetext{
14 Entre ellas las categorías de crítica técnica, crítica conceptual, utilización de datos (como caja negra), etc.
}

a los resultados que en Argentina. Esto es debido a que en nuestro país, la prueba PISA se ha cajanegrizado, es decir, se ha vuelto tan exitosa y validada que sólo se presta atención a los resultados de salida, sin atender a los distintos elementos que la constituyen y que a su vez van constituyendo esos resultados. Contrariamente en Argentina, se ha abierto la prueba PISA y explorado, entendiéndola como una construcción sociopolítica no neutral sujeta a críticas.

Revisando las noticias se aprecia que en Chile no se cuestiona la visión de mundo o la teoría que construye a la prueba PISA, sólo se habla de sus resultados, asumiendo que muestran nuestra realidad educativa con objetividad. De este modo, es posible que los resultados obtenidos en la prueba PISA tengan un nivel performativo mayor en Chile que en Argentina.

A modo de ejemplo, cito algunas noticias que grafican lo anteriormente señalado. En el diario el Télam se lee que el Ministro de Educación Argentino, Alberto Sileoni, en el marco del $15^{\circ}$ congreso Mundial de Educación Comparada celebrado en Buenos Aires en junio del 2013, criticó los resultados argumentando que en la construcción de PISA no se han considerado las diferencias contextuales, poniendo de ese modo a los países invitados en desventaja ("Cuestionan una prueba", 2013).

En la misma línea, varios diarios Argentinos y Uruguayos, a diferencia de los Chilenos, mencionaron las críticas y recomendaciones elaboradas por los países miembros del MERCOSUR ${ }^{15}$ a la prueba PISA en el marco del Seminario Mercosur: Políticas de Evaluación Educativa en la Región, que se celebró en Buenos Aires el 07 de junio de 2012, y en la XIX reunión de ministros de educación del MERCOSUR, celebrado el 21 de junio de 2013 en Montevideo, Uruguay. Entre las críticas se destaca el hecho de que los países de la OCDE cuentan con una realidad más homogénea que la de América Latina, y que no se han considerado en la elaboración de la prueba las particularidades de los contextos latinoamericanos (La crítica oficial, 2013; El MERCOSUR pidió, 2013).

Durante el mismo período, en Chile no se señaló en prensa electrónica la prueba PISA, ni las críticas señaladas en diarios Argentinos. En nuestro país se observaron algunas opiniones que no hacían eco con los cuestionamientos enunciados en Argentina, sino que más bien mostraban una validación de la prueba, que desde su concepción, estaba mostrando en términos

\footnotetext{
15 Mercado Común del Sur, cuyos países miembros son Argentina, Bolivia, Uruguay, Paraguay, Venezuela y Brasil.
} 
objetivos el progreso chileno en términos de calidad educativa.

Por medio del seguimiento de las noticias, ha sido posible seguir de manera empírica algunos de los rastros que ha ido dejando la prueba PISA en su recorrido, y ver a partir de ellos, que pese a la intencionalidad de la OCDE, la prueba PISA termina actuando de manera diferente en cada país según el modo en que los demás actantes interactúen con ella, y no de manera estándar como se sostiene desde la organización, en base al apego de los protocolos de aplicación y corrección que han diseñado y que demandan seguir a las partes involucradas en el proceso de operacionalización.

Por medio de este ejercicio quedó de manifiesto que la prueba PISA cuenta con una mayor validación en Chile que en Argentina, es decir, se obtuvo un hallazgo que de seguirlo, nos continuaría mostrando la complejidad de la prueba PISA y sus múltiples recorridos. Como en Chile se le concede a la prueba PISA una calidad diagnóstica y prescriptiva mayor que en Argentina, se puede esperar que en nuestro país la prueba PISA cuente con una mayor agencia regulativa o de orientación para la formulación de políticas educativas, cuestión que, por lo demás, es uno de los objetivos con que fue creada (Gorur, 2011).

\section{Conclusión}

Por medio de la discusión y de los ejercicios de desplazamiento epistémico desplegados, es posible ver que una mirada alternativa de la prueba PISA, basada en la comprensión de la misma, contribuye a tener una visión reticular más comprehensiva y por ello menos sesgada del dispositivo en sí.

Al abrir PISA y someterla a un análisis constructivista, cobra sentido problematizar la dicotomía asimétrica entre sujeto-objeto (donde se le atribuye al segundo objetividad y con ello de manera agregada validez), porque al seguir el proceso de su construcción y sus prácticas se evidencia que un dispositivo como la prueba PISA no es sólo un dispositivo técnico aislado de procesos sociales, políticos e ideológicos, sino que más bien es un constructo imbricado y múltiple en el que todas esas dimensiones son parte constitutiva de la prueba en sí. En términos epistémicos entonces, es posible entrar en el entramado de discusión en torno a PISA, planteando que su concepción como un dispositivo neutral, validado por los tecnicismos que la construyen al margen de lo social, político y económico es una especie de ilusión propia de la epistemología moderna mecanicista.

Finalmente, quisiera sostener que los ejercicios de desplazamiento epistémico de la prueba PISA sirven a una mejor comprensión de ella, al otorgar una perspectiva diferente que logra problematizarla, contribuyendo de ese modo al estado de la discusión en torno a la prueba al ofrecer herramientas para ponderarla de manera más cauta, teniendo presente su agencia y con ello su impacto en nuestras realidades educativas.

\section{Referencias}

Bijker, W. (1997). Of bicycles, bakelites, and bulbs: Toward a theory of sociotechnical change. Cambridge: MIT Press.

Bodin, A. (2007). What Does PISA Really Assess? What Does It Not? A French View. En G. Brinek, S. Hopmann \& M. Retzl (Eds.), PISA According to PISA - Does PISA Keep What It Promises? (pp. 21-55). New Brunswick: Transaction Publishers.

Brinek, G. \& Hopmann, S. (2007). Introduction: PISA according to PISA. Does PISA Keep What It Promises?. En G. Brinek, S. Hopmann \& M. Retzl (Eds.), PISA According to PISA - Does PISA Keep What It Promise? (pp. 9-19). New Brunswick: Transaction Publishers.

Callon, M. \& Law, J. (1998). De los intereses y su transformación. Enrolamiento y contraenrolamiento. En M. Domènech \& F. Tirado (Eds.), Sociología simétrica. Ensayos sobre ciencia, tecnología y sociedad (pp. 51-62). Barcelona: Gedisa.

Consejo Latinoamericano de Ciencias Sociales (2014). No a PISA. Por una evaluación al servicio de una educación emancipadora. Recuperado de http://www.clacso.org.ar/difusion/ Declaracion_GT_politicas_educativas/Declaracion_GT_Politicas_educativas_y_derecho_a_la_educacion_en_America_Latina_y_El_Caribe.pdf

Cuestionan una prueba de OCDE por considerar a la escuela como productora de mano de obra. (23 de junio de 2013). Télam. Recuperado de http://www.telam.com.ar/notas/201306/21432cuestionan-una-prueba-de-ocde-por-considerara-a-las-escuelascomo-productora-de-mano-de-obra.html

Dolin, J. (2007). PISA - An Example of the Use and Misuse of Large-Scale Comparative Test. En G. Brinek, S. Hopmann \& M. Retzl (Eds.), PISA According to PISA - Does PISA Keep What It Promises? (pp. 93-125). New Brunswick: Transaction Publishers.

El MERCOSUR pidió criterios regionales de evaluación para la prueba PISA. (17 de marzo de 2013). Télam. Recuperado de http://www.telam.com.ar/notas/201303/10659-el-mercosur-pidiocriterios-regionales-de-evaluacion-para-la-prueba-pisa.html

Falabella, A. (2014). The Performing School: The Effects of Market \& Accountability Policies. Education Policy Analysis Archives, 22(70), 1-29. doi:10.14507/epaa.v22n70.2014

Foucault, M. (2002). La Hermenéutica del Sujeto. Curso en el College de France. (1981-1982). México: Fondo de Cultura Económica.

Foucault, M. (2005). El Poder Psiquiátrico. Curso en el College de France. (1973-1974). México: Fondo de Cultura Económica.

Foucault, M. (2006). Seguridad, Territorio, Población. Curso en el College de France. (1977-1978). Buenos Aires: Fondo de Cultura Económica.

Foucault, M. (2007). Nacimiento de la Biopolítica. Curso en el College de France. (1978-1979). México: Fondo de Cultura Económica. 
Foucault, M. (2009). El Gobierno de si y de los otros. Curso en el College de France. (1982-1983). México: Fondo de Cultura Económica.

Foucault, M. (2012). Vigilar y Castigar, nacimiento de la prisión. Buenos Aires: Siglo Veintiuno Editores.

Gorur, R. (2011). ANT on the PISA Trail: Following the statistical pursuit of certainty. Educational Philosophy and Theory, 43(s1), 76-93. doi:10.1111/j.1469-5812.2009.00612.x

Haraway, D. (1988). Situated Knowledges: The Science Question in Feminism and the Privilege of Partial Perspective. Feminist Studies, 14(2), 575-599. doi:10.2307/3178066

Haraway, D. (1989). Primate Visions: Gender, Race, and Nature in the World of Modern Science. New York: Routledge.

Haraway, D. (1991). Ciencia, cyborgs y mujeres. La reinvención de la naturaleza. Londres: Free Association Books.

Knorr, K. (1995). Laboratory Studies: The Cultural Approach to the Study of Science. En S. Jasanoff, G. Markle, J. Petersen \& T. Pinch (Eds.), Handbook of Science and Technology Studies (pp.140-166). Londres, Inglaterra: Sage.

La crítica oficial a una prueba educativa genera polémica ( 26 de junio de 2013). Clarín. Recuperado de http://www.clarin.com/sociedad/ critica-oficial-prueba-educativa-polemica_0_945505508.html

Latour, B. (1998). La tecnología es la sociedad hecha para que dure. En M. Domènech, \& F. Tirado (Eds.), Sociología Simétrica. Ensayos sobre ciencia, tecnología y sociedad (pp. 109-142). Barcelona: Gedisa.

Latour, B. (1999). La esperanza de Pandora. Ensayos sobre la realidad de los estudios de la ciencia. Barcelona: Gedisa.

Latour, B. (2001). La esperanza de Pandora. Barcelona: Gedisa.

Latour, B. (2005). Reensamblar lo Social. Una introducción a la teoría del actor-red. Buenos Aires: Manantial.

Latour, B. (2007). Nunca Fuimos Modernos, Ensayo de antropología simétrica. Buenos Aires: Siglo XXI.
Leach, M., Bloom, G., Ely, A., Nightingale, P., Scoones, I., Shah, E. \& Smith, A. (2007). Understanding governance: pathways to sustainability, STEPS Working Paper 2. Recuperado de http:// steps-centre.org/wp-content/uploads/final_steps_governance.pdf

Leibfried, S. \& Martens, K. (2009). PISA: internacionalización de la política educativa o ¿cómo se llega de la política nacional a la OCDE?. Profesorado. Revista de Currículum y Formación de Profesorado, 12(2), 1-11. Recuperado de http://www.ugr. es/ recfpro/rev132ART9.pdf

OCDE and PISA tests are damaging education worldwide - academics. (6 de mayo de 2014). The Guardian. Recuperado desde: http:// www.theguardian.com/education/2014/may/06/oecd-pisa-testsdamaging-education-academics

Organización para la Cooperación y el Desarrollo Económico (2014). Perspectivas económicas de América Latina 2015 Educación, competencias e innovación para el desarrollo. doi:10.1787/ leo-2015-es

Organización para la Cooperación y el Desarrollo Económico. $L a$ $O C D E$. ¿Qué es la OCDE? Recuperado de http://www.oecd.org/ centrodemexico/laocde/

Organización para la Cooperación y el Desarrollo Económico. (2006). Informe PISA 2006, Marco de la Evaluación. Conocimientos y habilidades en Ciencias, Matemáticas y Lectura. Recuperado de http://www.agenciaeducacion.cl/wp-content/files_mf/ marco_de_evaluacion_pisa_2006_en_espanol.pdf

Pedró, F. (2012). Deconstructing PISA's Bridges: From Results Analysis to Political Prescriptions. Revista española de educación comparada, 19, 139-172. doi:10.5944/reec.19.2012.7581

Puchhammer, M. (2007). Language-Based Item Analysis - Problems in Intercultural Comparisons. En G. Brinek, S. Hopmann \& M. Retzl (Eds.), PISA According to PISA - Does PISA Keep What It Promises? (pp. 127-137). New Brunswick: Transaction Publishers. 